\title{
A New Monotone Fuzzy Rule Relabeling Framework With Application to Failure Mode and Effect Analysis Methodology
}

\author{
LIE MENG PANG ${ }^{\circledR 1}$, (Member, IEEE), KAI MENG TAY ${ }^{\circledR 2}$, CHEE PENG LIM ${ }^{\circledR 3}$, \\ AND HISAO ISHIBUCHI ${ }^{\circledR}$, (Fellow, IEEE) \\ ${ }^{1}$ Guangdong Provincial Key Laboratory of Brain-Inspired Intelligent Computation, Department of Computer Science and Engineering, Southern University of \\ Science and Technology, Shenzhen 518055, China \\ ${ }^{2}$ Faculty of Engineering, Universiti Malaysia Sarawak, Kota Samarahan 94300, Malaysia \\ ${ }^{3}$ Institute for Intelligent Systems Research and Innovation, Deakin University, Waurn Ponds, VIC 3216, Australia \\ Corresponding author: Hisao Ishibuchi (hisao@sustech.edu.cn)
}

This work was supported in part by the National Natural Science Foundation of China under Grant 61876075, in part by the Guangdong Provincial Key Laboratory under Grant 2020B121201001, in part by the Program for Guangdong Introducing Innovative and Entrepreneurial Teams under Grant 2017ZT07X386, in part by the Shenzhen Science and Technology Program under Grant KQTD2016112514355531, and in part by the Program for the University Key Laboratory of Guangdong Province under Grant 2017KSYS008.

\begin{abstract}
A monotone fuzzy rule relabeling (MFRR) algorithm has been introduced previously for tackling the issue of a non-monotone fuzzy rule base in the Takagi-Sugeno-Kang (TSK) Fuzzy Inference System (FIS). In this paper, we further propose a new three-stage framework to develop a computationally efficient MFRR algorithm. The first stage determines the combinations of fuzzy rules to be relabeled by exploiting the prior information derived from a given non-monotone fuzzy rule base. This prior information includes the minimum number of fuzzy rules to be relabeled (denoted as $k$ ), as well as the states of fuzzy rules that must be, must not be, or may be relabeled. The second stage relabels the consequent parts of multiple sets of $k$ noisy fuzzy rules obtained from the first stage, such that a monotone fuzzy rule base is produced. The third stage selects the most suitable relabeled fuzzy rule base among the potential monotone fuzzy rule bases obtained from the second stage, either objectively or subjectively. We provide insights into MFRR and discuss its practical implementation. In addition, a network flow method is fused with the proposed MFRR framework, resulting in an efficient computation scheme. The MFRR framework is applied to Failure Mode and Effect Analysis (FMEA) problems related to a sewage treatment plant and a public hospital. It is also evaluated with real FMEA information from a semiconductor plant. The results are analyzed and discussed, which positively demonstrate the effectiveness of the proposed MFRR framework in formulating a monotone TSK-FIS model for undertaking FMEA problems.
\end{abstract}

INDEX TERMS Fuzzy inference systems, failure mode and effect analysis, graph theory, monotonicity, monotone fuzzy rule relabeling, network flow method.

\section{INTRODUCTION}

Fuzzy inference systems (also known as fuzzy rule-based systems, fuzzy logic systems, or fuzzy systems) are one of the most successful applications of fuzzy set theory [1]. The fuzzy inference systems can be regarded as universal approximators. This means by using a set of fuzzy If-Then rules and an appropriate inference mechanism, a fuzzy inference

The associate editor coordinating the review of this manuscript and approving it for publication was Shun-Feng $\mathrm{Su}^{\text {(D) }}$. system can approximate any continuous real function with arbitrary accuracy defined in a compact domain [2]. The fuzzy inference systems are also well known for their ability to handle imprecise information and model complex and non-linear systems [3]. For these reasons, the fuzzy inference systems have become a popular computing approach, and have been used in many successful applications across different fields [3], [4].

Monotone fuzzy inference systems [5], [6] are a class of fuzzy systems that requires a monotonicity relationship 